\title{
DOME-SHAPED EUV WAVES FROM ROTATING ACTIVE REGIONS
}

\author{
M. Selwa ${ }^{1}$, S. Poedts ${ }^{1}$, And C. R. DeVore ${ }^{2}$ \\ ${ }^{1}$ Centre for Plasma Astrophysics, Department of Mathematics, K.U. Leuven, Celestijnenlaan 200 B, 3001 Leuven, Belgium; \\ mag.selwa@wis.kuleuven.be, stefaan.poedts@wis.kuleuven.be \\ 2 Naval Research Laboratory, 4555 Overlook Avenue SW, Washington, DC 20375, USA; devore@lcp.nrl.navy.mil \\ Received 2011 October 27; accepted 2012 February 2; published 2012 February 16
}

\begin{abstract}
Recent STEREO observations enabled the study of the properties of EUV waves in more detail. They were found to have a three-dimensional (3D) dome-shaped structure. We investigate, by means of 3D MHD simulations, the formation of EUV waves as the result of the interaction of twisted coronal magnetic loops. The numerical simulation is initialized with an idealized dipolar active region and is performed under coronal (low $\beta$ ) conditions. A sheared rotational motion is applied to the central parts of both the positive and negative flux regions at the photosphere so that the flux tubes in between them become twisted. We find that the twisting motion results in a dome-shaped structure followed in space by a dimming and in time by an energy release (flare). The rotation of the sunspots is the trigger of the wave which initially consists of two fronts that later merge together. The resulting EUV wave propagates nearly isotropically on the disk and $\sim 2$ times faster in the upward direction. The initial stage of the evolution is determined by the driver, while later the wave propagates freely with a nearly Alfvénic speed.
\end{abstract}

Key words: Sun: corona - Sun: flares - sunspots - waves

Online-only material: color figures

\section{INTRODUCTION}

The connection of the photosphere to the corona is an important factor determining the dynamics of coronal events. A possible source of coronal flows and motions may come from the dynamics of sunspots, e.g., their rotation. Rotating sunspots have been widely studied in the past century. The first observational evidence of photospheric twisting motions was found by Kempf (1910) and Evershed (1910). Yan et al. (2008) classified the active regions (ARs) in terms of rotation configuration and resulting flares.

EUV waves are often associated with solar flares. Biesecker et al. (2002) found that $66 \%$ of the EUV waves are associated with solar flares. Recently, Schrijver et al. (2011) found with SDO/AIA $211 \AA$ data that the major solar flare on 2011 February 2 originating from NOAA 11158 did not trigger the EUV wave, but started only once the wave traveled away a small distance. It is noteworthy that NOAA 11158 consisted of five sunspots that rotated/spinned $50^{\circ}-130^{\circ}$ (some clockwise, some counterclockwise) over five days of observations and released more than 40 smaller flares in addition to the main X-class flare of 2011 February 2 (Brown 2011). There are several suggestions regarding the origin and nature of EUV waves: a flare-initiated blast wave, e.g., Warmuth et al. 2004; a coronal mass ejection (CME) related event, e.g., Cliver et al. 2005, Chen 2006; EUV waves resulting from the generation of electric currents while considering the connectivity change during the CME eruption (Delannée et al. 2007, 2008). The nature of EUV waves also remains unclear: it might be fast mode waves (Wang 2000; Patsourakos et al. 2009), a phenomenon produced by the magnetic field restructuring during the CME eruption (Delannée et al. 2007), or a soliton (Wills-Davey et al. 2007).

Since the launch of STEREO there has been interest in the three-dimensional (3D) structure of the EUV waves. Patsourakos \& Vourlidas (2009) used forward modeling of the CME and the EUV wave and found that the best fit a CME is different from a best-fit EUV wave. The best-fit wave reveals the dome-like shape (see their Figure 4). Recently, Veronig et al. (2010) reported for the first time the 3D dome-like structure of EUV waves (see their Figure 2). It is noteworthy that such a dome propagates upward twice faster than on the disk (Veronig et al. 2010 reported the speed of upward propagation as $650 \mathrm{~km} \mathrm{~s}^{-1}$ while the speed of the wave on the disk is $280 \mathrm{~km} \mathrm{~s}^{-1}$ ). Figure 1 shows that the event of 2011 February 2 also has a dome-shape structure.

Due to their complexity, EUV waves have so far not been widely studied numerically in three dimensions. On the other hand, there are many 2D studies; e.g., Pomoell et al. (2008) studied the EIT waves created by a lift-off of a filament, while Chen et al. (2002) showed that a piston-driven shock produces Moreton waves and slower moving features preceding an enhanced plasma region which could correspond to EUV waves. More recently, Wang et al. (2009) proposed a different mechanism in a 2D study of shock formation leading to an EUV wave: rapid motions of the flux tube following the velocity vortices. The few full 3D simulations usually focus on the disk-observed part of the EUV wave, not mentioning its 3D structure, e.g., Wu et al. (2001), used the global magnetic field from a Wilcox Solar Observatory (WSO) magnetogram extrapolation and gravitationally stratified density to show that EUV waves as fast magnetosonic waves. Ofman \& Thompson (2002) and Ofman (2007) studied the interaction of EUV waves with dipolar ARs. The authors found that the waves undergo strong refraction and reflection. More recently, Schmidt \& Ofman (2010) presented the first 3D MHD study of an EUV wave triggered by a CME.

The goal of this Letter is to study, by means of numerical simulations, the 3D structure of the EUV waves as well as to propose a new excitation mechanism related to rotating sunspots to extend the current studies supporting only velocity or pressure pulses or a CME as a trigger of EUV waves. This Letter is organized as follows: the numerical model is described in Section 2, and the numerical results are presented and discussed in Section 3. This Letter is concluded by a presentation of the main results in Section 4. 

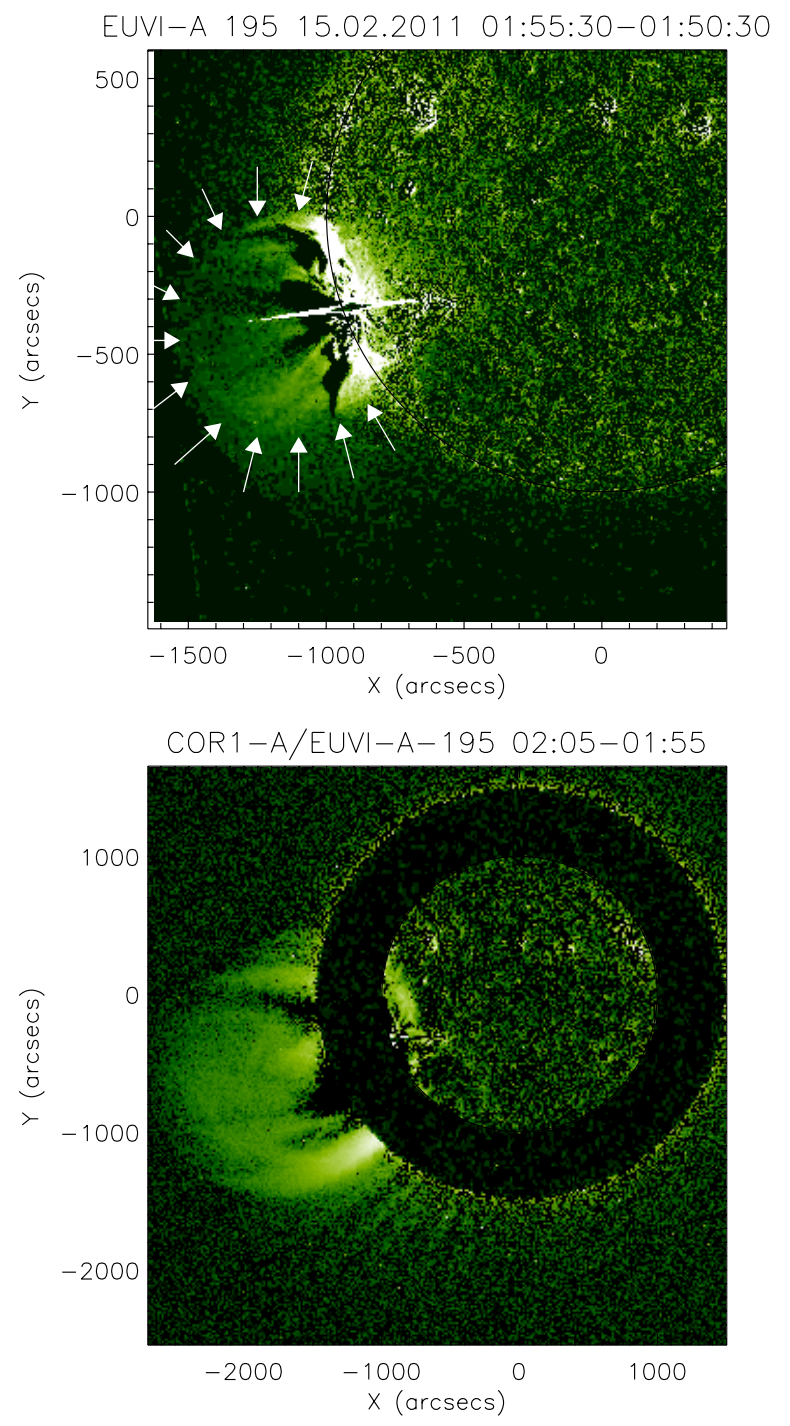

Figure 1. Top panel: STEREO/EUVI-A $195 \AA 5$ minute running difference image of the wave dome zoomed around NOAA11158. Arrows outline the wave dome. Bottom panel: composite of STEREO/EUVI-A $195 \AA$ and STEREO/COR1-A 10 minute running difference image (note the larger FOV and later time compared to the top panel).

(A color version of this figure is available in the online journal.)

\section{NUMERICAL MODEL}

We describe solar plasma with the $3 \mathrm{D}$ resistive nonlinear MHD equations:

$$
\begin{gathered}
\frac{\partial \varrho}{\partial t}+\nabla \cdot(\varrho \mathbf{V})=0 \\
\frac{\partial \varrho \mathbf{V}}{\partial t}+\nabla \cdot(\varrho \mathbf{V V})+\nabla P-\frac{1}{\mu}(\nabla \times \mathbf{B}) \times \mathbf{B}=0 \\
\frac{\partial U}{\partial t}+\nabla \cdot(U \mathbf{V})+P \nabla \cdot \mathbf{V}-\frac{\eta}{\mu}(\nabla \times \mathbf{B})^{2}=0 \\
\frac{\partial \mathbf{B}}{\partial t}-\nabla \times(\mathbf{V} \times \mathbf{B})+\nabla \times(\eta \nabla \times \mathbf{B})=0
\end{gathered}
$$

Here, $\mathbf{V}$ denotes the velocity field, $\mathbf{B}$ is the magnetic field, $\varrho$ is the mass density, $U$ is the internal energy density, $P$ is the plasma pressure, $\mu$ is the magnetic permeability, and $\eta$ is the magnetic diffusion coefficient. The internal energy and pressure are related via $U=P /(\gamma-1)$, where $\gamma$ is the ratio of specific heats. We perform our simulations in scaled dimensionless units, setting $\gamma=5 / 3$ and $\mu=4 \pi$, and the gas constant to the scaled value $R=0.01$. Equations (1)-(4) are solved in resistive MHD regime $\left(\eta=10^{-4}\right)$.

We start our simulation with a dipolar magnetic field and a uniform mass density field and temperature. Scaled initial values of density $\varrho=1$ and pressure $p=0.001$ were chosen yielding an initial temperature $T=0.1$ and sound speed $c_{s} \simeq 0.04$. Additionally, the characteristic strength of the dipole described by the scalar potential $\phi$ and oriented along the $y$-axis at depth $d=2$ is $|B|=100$ :

$$
\phi(x, y, z)=|B| d^{3} \frac{y}{\left[(x+d)^{2}+y^{2}+z^{2}\right]^{3 / 2}} .
$$

The perturbation of the system is applied at the bottom boundary with the velocity field, $\mathbf{V}(x, y, 0, t)$, that we use to drive the coronal system. The driving boundary velocities were constant in time and linearly dependent on the strength of the normal component of magnetic field, $\left|B_{n}\right|$ :

$$
\mathbf{V}=1.2\left(\hat{\mathbf{n}} \times \nabla B_{n}\right) \begin{cases}0 & \text { for }\left|B_{n}\right| \leqslant B_{-}, \\ \left(\left|B_{n}\right|-B_{-}\right) /\left(B_{+}-B_{-}\right) & \text {for } B_{-} \leqslant\left|B_{n}\right| \leqslant B_{+}, \\ 1 & \text { for } B_{+} \leqslant\left|B_{n}\right| .\end{cases}
$$

We applied rotation to the central region of the dipolar spots ( $B_{-}=20$ and $B_{+}=50$ ), so strong-field regions rotate while the weak-field regions are left unaltered.

The initial magnetic field at the base of the simulation region together with the flow pattern is shown in Figure 2.

\section{NUMERICAL RESULTS}

Equations (1)-(4) are solved numerically with the ARMS code (DeVore 1991; Welsch et al. 2005) in an Eulerian box with the $x$-, $y$-, and $z$-dimensions $\left(x_{\min }, x_{\max }\right) \times\left(y_{\min }, y_{\max }\right) \times$ $\left(z_{\min }, z_{\max }\right)=(0,2 L) \times(-L, L) \times(-L, L)$, where $L=9$ and, therefore, the Alfvén time $\tau_{A}=L / \max \left(V_{A}\right) \simeq 0.19$. Here, the $x$-direction is the vertical direction and the dipolar field extends vertically in the $x$-direction from the $y-z$ plane. The numerical box is covered with $128^{3}$ grid points. Grid convergence studies showed that this resolution is sufficient to get results independent of the grid. Extrapolative, zero-gradient, closed boundary conditions (zero flux) of all plasma variables are applied at all the boundaries, preventing flux loss through the boundaries.

The twisting motion of the dipole leads to the initiation of a global coronal wave. The resulting wave propagates almost isotropically in the lateral directions (middle panel of Figure 3) and has a dome-like shape seen in line of sight (LOS) integrated $\varrho^{2}$ at the limb (bottom panel of Figure 3) similar to the one observed by Patsourakos \& Vourlidas (2009) and Veronig et al. (2010).

The global coronal wave is initialized as a result of the shearing motion. Initially, the plasma is pushed away from the center of the dipole and forms plasma concentrations-brighter points and depletions - subsequently forming a coronal dimming following the wave (not shown). Later, two wave fronts are formed that propagate away from the dipole (top panel of Figure 3) and merge together into an isotropic wave (middle panel of Figure 3). A similar behavior of two initiation centers of EUV wave followed by a single circular front were observed by Temmer et al. (2011; see their Figure 1). 

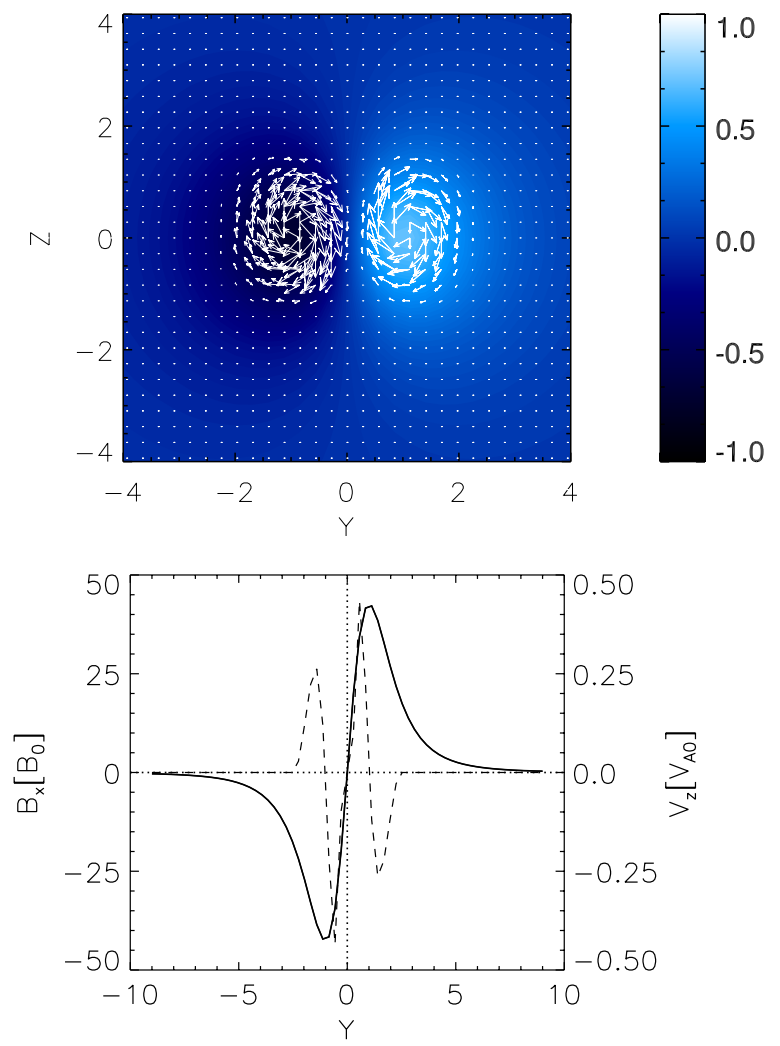

Figure 2. Top panel: bottom boundary of the initial state of simulation: normal magnetic field component (color scale) and velocity (arrows). Values of normal component of magnetic field are given in units of an initial maximum normal component of magnetic field in the system, $B_{0 n}=42$. Bottom panel: a cut along the $x=z=0$ line showing the normal component of the magnetic field (solid line) together with the corresponding velocity of the driver (dashed line) normalized to maximum initial values.

(A color version of this figure is available in the online journal.)

Figure 4 shows the magnetic and kinetic energies during the simulation. The first major kinetic energy release (around $t \sim 1 \tau_{A}$, where $\tau_{A}$ is the Alfvén time) corresponding to a decrease of magnetic energy (flare) occurs after the wave was initialized.

The top panel of Figure 5 shows the propagation distance of the wave versus time. It is measured by fitting the Gaussian function into the $x$-, $y$-, or $z$-cut of the LOS-integrated mass density. It is clear that the wave propagates faster in the upward direction than in the lateral direction $(\sim 2.25$ times for the $y$-direction and $\sim 1.5$ times for the $z$-direction). A similar behavior was observed by Veronig et al. (2010) with a velocity ratio of $\sim 2.3$. The bottom panel of Figure 5 presents the velocity of the wave in all three axial directions together with the mean Alfvén speed at each location. The calculation of the speed of the wave is based on the propagation distance according to the central difference scheme. We use every third point on each side in order to get a relatively smooth curve. The "mean Alfvén speed at each location" refers to the Alfvén speed corresponding to the considered point on the disk $(y-z)$ plane and averaged in the vertical $(x)$ direction to the height of the dome. We clearly see that the wave propagates at Alfvénic (slightly super-Alfvénic) speed which indicates a fast magnetosonic wave. It is noteworthy that at the initial stage of the wave evolution the gradient of the propagation distance (i.e., the velocity) is different from that in the later stage. This is due to the fact that in the beginning the wave is still driven by a driver (rotation of the sunspots resulting in the expanding twisted loops) while later it propagates freely
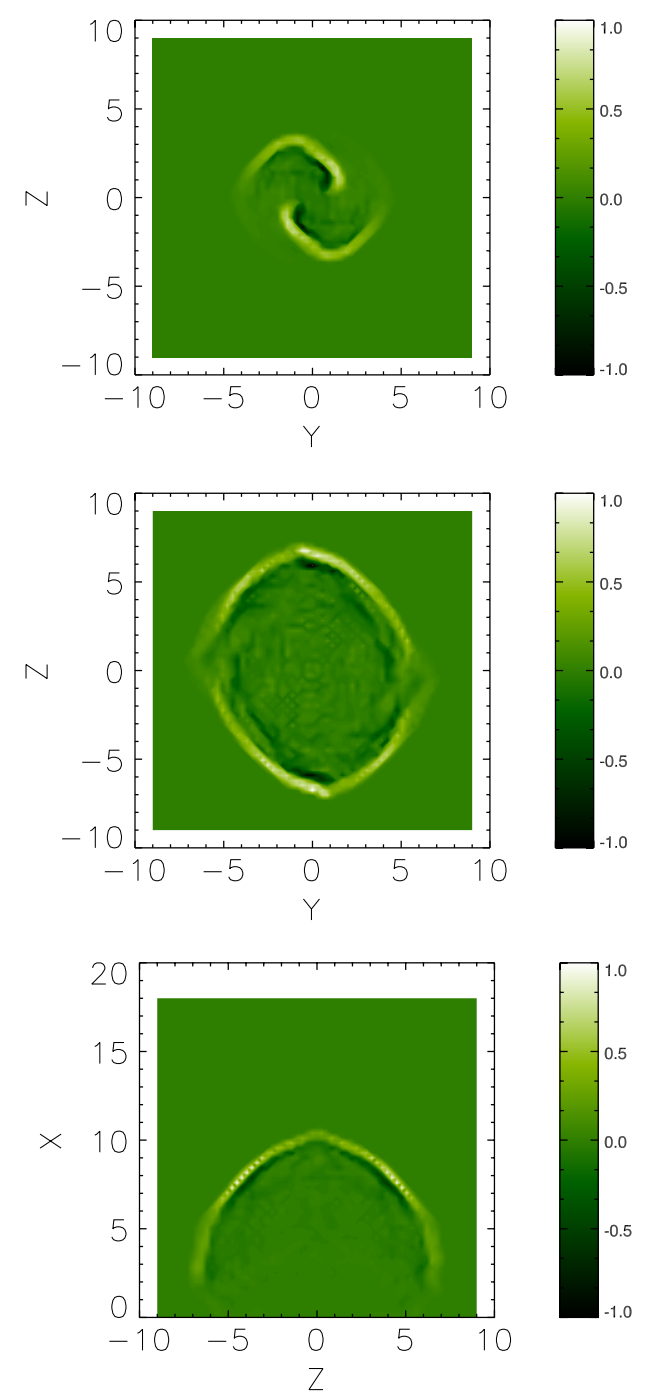

Figure 3. Difference images of the LOS-integrated $\varrho^{2}$ on the disk at $t=0.75 \tau_{A}$ (top panel) and $t=2.5 \tau_{A}$ (middle panel) and at the limb at $t=2.5 \tau_{A}$ (bottom panel). Values of LOS-integrated $\varrho^{2}$ are given in units of $20 \cdot \max \left(\varrho_{0}^{2}\right)$ (top panel), $40 \cdot \max \left(\varrho_{0}^{2}\right)$ (middle panel), and $99 \cdot \max \left(\varrho_{0}^{2}\right)$ (bottom panel). (A color version of this figure is available in the online journal.)

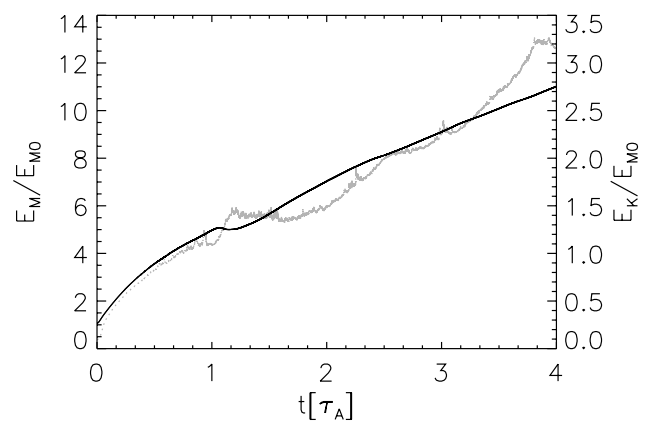

Figure 4. Temporal evolution of the magnetic (black solid line) and the kinetic (gray dotted line) energies. Values of energy are given in units of an initial magnetic energy in the system.

as a fast magnetoacoustic wave. Similar examples are observed on the Sun when in the initial stage of evolution the wave and the driver (most often an expanding CME) are still coupled (e.g., Patsourakos \& Vourlidas 2009; see their Figure 3). Additionally, the acceleration of the wave changes sign after the initial phase: at first, the wave accelerates while at the later stage corresponding to the free propagation of the wave, it decelerates. 

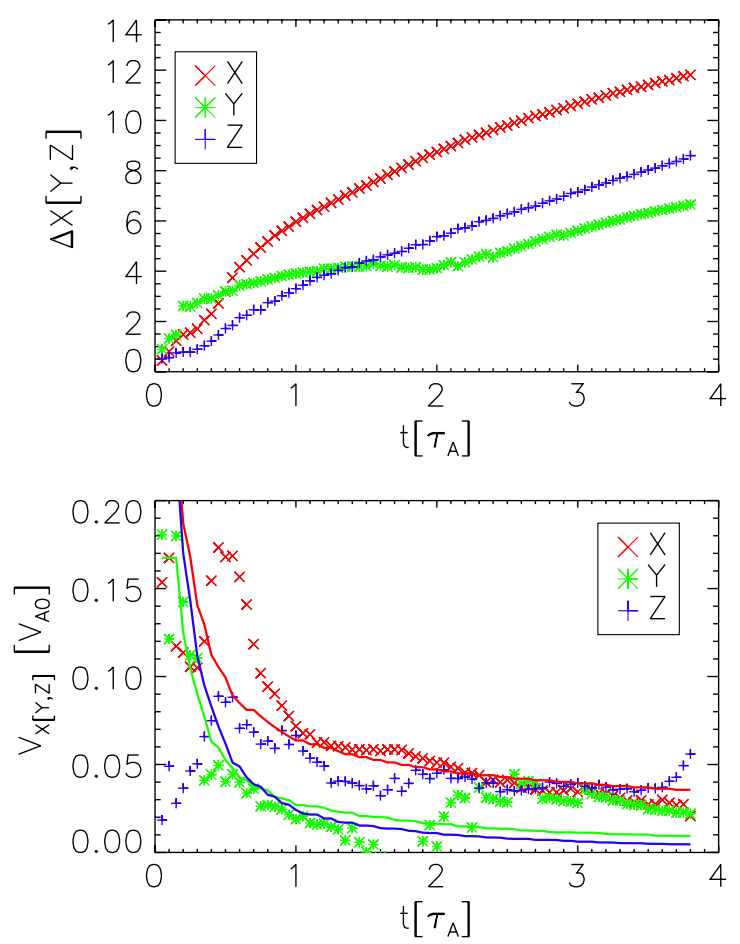

Figure 5. Top panel: propagation distance of the EUV wave vs. time. Bottom panel: wave speed vs. time. The solid lines show the mean Alfvén speed at the distance where the wave moved to. The red $\times$ signs correspond to the vertical propagation while green $*$ and blue + signs show the propagation on the disk. (A color version of this figure is available in the online journal.)

Although the speed of the rotation is quite high in our simulation, we checked that the wave is present in the system independent of the rotation speed of the driver. Only the wave speed in the initial evolution stage is determined by the driver. As a matter of fact, later on once the wave decouples from the driver, the perturbation propagates freely with its own Afvénic speed as a fast magnetoacoustic wave.

One drawback of our model is the lack of gravity in the system. A gravitationally stratified atmosphere would make our model (mainly the Alfvén velocity profile) more realistic and we plan to add it in our future work. However, as the described simulation corresponds to the initial stage of the wave evolution (close to the AR) and does not involve global corona modeling, we decided to use this simple model in which the Alfvén speed profile is decreasing with height, as is observed in ARs; see, e.g., Verwichte et al. (2010). Additionally, since the coronal density scale is $\sim 100 \mathrm{Mm}$, any density effects on the wave propagation will be small except quite high above the surface, i.e., once the wave moves further away from the AR.

\section{SUMMARY}

The observations show the presence of EUV waves originating from rotating ARs. The EUV waves are often (66\%) associated with flares (Biesecker et al. 2002). Some of the observed waves have a dome-like shape (Patsourakos \& Vourlidas 2009; Veronig et al. 2010). We presented another example of a dome-shaped EUV wave originating from a rotating AR (NOAA11158).

We model EUV waves originating from rotating sunspots in ARs. We use a 3D resistive MHD model of a dipolar AR with a constant density under coronal (low plasma $\beta$ ) conditions. We find that the trigger of the wave is the shearing motion of the sunspots that results in density enhancements (forming the bright front) and depletions (forming a coronal dimming). Only after the wave starts to propagate, we observe an energy release (flare), which is in agreement with the events reported by Kienreich et al. (2009) and Schrijver et al. (2011). The global coronal wave seen in emission seems to propagate nearly isotropically on the solar disk. The wave also propagates in the upward direction, forming a dome-like shape. The upward propagation is $\sim 2$ times faster than the lateral propagation, which agrees with the observations for the dome-like EUV wave (Veronig et al. 2010). The initial stage of the wave evolution is determined by the driver (in agreement with Patsourakos et al. 2009). We observe two initiation centers of the wave before it becomes one circular front (in agreement with the observations of Temmer et al. 2011).

M.S. expresses thanks to Dr. S. Patsourakos for helpful discussions. The authors thank the unknown referee for the constructive comments that helped to improve the Letter. M.S.'s work was supported by the SIDC Data Exploitation (ESA Prodex 9) project. Part of her work was supported by the European Commission under the Solaire Marie Curie Training Network. S.P.'s work was supported by the projects GOA/2009-009 (K.U. Leuven), G.0729.11 (FWO-Vlaanderen), and SIDC Data Exploitation (ESA Prodex 9). Financial support by the European Commission through the SOTERIA (Collaborative project 218816 of FP7-SPACE-2007-1) is gratefully acknowledged as well as support from the European Commission's Seventh Framework Programme (FP7/2007-2013). C.R.D.'s work was supported by NASA.

\section{REFERENCES}

Biesecker, D. A., Myers, D. C., Thompson, B. J., Hammer, D. M., \& Vourlidas, A. 2002, ApJ, 569, 1009

Brown, D. 2011, Rotating Sunspots Spin Up a Super Solar Flare, PHYSorg.com, 2011 April 21, http://www.physorg.com/news/2011-04-rotating-sunspotssuper-solar-flare.html

Chen, P. F. 2006, ApJ, 641, L153

Chen, P. F., Wu, S. T., Shibata, K., \& Fang, C. 2002, ApJ, 572, L99

Cliver, E. W., Laurenza, M., Storini, M., \& Thompson, B. J. 2005, ApJ, 631, 604

Delannée, C., Hochedez, J. F., \& Aulanier, G. 2007, A\&A, 465, 603

Delannée, C., Török, T., Aulanier, G., \& Hochedez, J. F. 2008, Sol. Phys., 247, 123

DeVore, C. R. 1991, J. Comput. Phys., 92, 142

Evershed, J. 1910, MNRAS, 70, 217

Kempf, P. 1910, Astron. Nachr., 185, 197

Kienreich, I. W., Temmer, M., \& Veronig, A. M. 2009, ApJ, 703, L118

Ofman, L. 2007, ApJ, 655, 1134

Ofman, L., \& Thompson, B. J. 2002, ApJ, 574, 440

Patsourakos, S., \& Vourlidas, A. 2009, ApJ, 700, L182

Patsourakos, S., Vourlidas, A., Wang, Y. M., Stenborg, G., \& Thernisien, A. 2009, Sol. Phys., 259, 49

Pomoell, J., Vainio, R., \& Kissmann, R. 2008, Sol. Phys., 253, 249

Schmidt, J. M., \& Ofman, L. 2010, ApJ, 713, 1008

Schrijver, C. J., Aulanier, G., Title, A. M., Pariat, E., \& Delannée, C. 2011, ApJ, 738,167

Temmer, M., Veronig, A. M., Gopalswamy, N., \& Yashiro, S. 2011, Sol. Phys., 273,421

Veronig, A. M., Muhr, N., Kienreich, I. W., Temmer, M., \& Vršnak, B. 2010, ApJ, 716, L57

Verwichte, E., Foullon, C., \& Van Doorsselaere, T. 2010, ApJ, 717, 458

Wang, H., Shen, C., \& Lin, J. 2009, ApJ, 700, 1716

Wang, Y. M. 2000, ApJ, 543, L89

Warmuth, A., Vršnak, B., Magdalenić, J., Hanslmeier, A., \& Otruba, W. 2004, A\&A, 418, 1117

Welsch, B. T., DeVore, C. R., \& Antiochos, S. K. 2005, ApJ, 634, 1395

Wills-Davey, M. J., DeForest, C. E., \& Stenflo, J. O. 2007, ApJ, 664, 556

Wu, S. T., Zheng, H., Wang, S., et al. 2001, J. Geophys. Res., 106, 25089

Yan, X. L., Qu, Z. Q., \& Kong, D. F. 2008, MNRAS, 391, 1887 